\title{
Clinico-epidemiological Study on Pesticide Poisoning in a Tertiary Care Hospital in Eastern Nepal
}

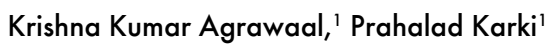 \\ 'Department of Internal Medicine, B. P. Koirala Institute of Health Sciences, Dharan, Nepal
}

\section{ABSTRACT}

Introduction: Pesticide poisoning is a major health problem worldwide. In Nepal the most common cause is suicidal and pesticides account for more than fifty percent of cases. The objective of the study was to look in detail regarding the pesticide poisoning cases admitted at BPKIHS; their epidemiological profile, presentation, treatment and their outcome during the hospital stay. Drug therapy included specific antidotal drugs; atropine and pralidoxime (PAM) and some nonspecificdrugs: antimicrobials and sedatives.

Methods: It was a retrospective study which included 2621 patients with poisoning of which 1661 cases were related to pesticides.

Results: The mean age at presentation was 29 years. The mean duration of hospital stay was 6.7 days. The majority of patients $81.16 \%$ showed improvement whereas $6.6 \%$ of patients died within 24 hours of admission and $3.54 \%$ after 48 hours of admission. Among all the patients $0.5 \%$ patients were given ICU care and all others were managed in the different units of medicine ward. The total amount of atropine administered varied considerably from patient to patient, according to the need. Most of the cases were under the influence of alcohol. All the patients had a psychiatry evaluation before discharge.

Conclusions: Pesticide poisoning is increasing in incidence and it is one of the preventable public health problems and includes mainly the patients' age group 20-30 years. Due to easy availability of pesticides it is the most preferred method of suicide, the main reasons being impulsive act and increased indebtedness in the society.

Keywords: pesticides; organophosphorous poisoning.

\section{INTRODUCTION}

Poisoning is one of the commonest causes of admission of young adults in the medical wards in Nepal. ${ }^{1}$ Organophosphorus (OP) compounds are used as pesticides, herbicides, and chemical warfare agents in the form of nerve gases (Sarin gas) and as therapeutic agents, such as ecothiopate used in the treatment of glaucoma. $2 ; 3$ Of the estimated 500,000 deaths from self-harm in the rural asia, each year about $60 \%$ are due to pesticide poisoning and studies estimate that organophosphorus pesticides are responsible

Correspondence: Dr Krishna Kumar Agrawaal, Department of Internal Medicine, B. P. Koirala Institute of Health Sciences, Dharan, Nepal. Email: agrawalkris@gmail.com, Phone: +977-9857045721. 
for around two-thirds of these deaths. ${ }^{4}$ In our part of world OP accounts for more than $75 \%$ of cases. ${ }^{5}$ The best-known effect of the OP compounds is inhibition of acetlycholinesterase (AChE), which causes the accumulation of acetylcholine in the body. However, OP compounds are also known to have effects on the GABA and glutamate systems, N-methyl-Daspartate (NMDA) receptors. ${ }^{6}$ The pesticide poisoning is one of the preventable public health problem in the developing countries. OP compounds are easily available for purchase in countries like Nepal where agriculture is the main industry with maximum land holdings for agriculture. ${ }^{7}$ The pesticides are available in ordinary shops and stored in improper manner due to lake of awareness of their hazard.Though there can be accidental poisoning due to inhalation or contact but most of the acute cases are due to deliberate self harm. ${ }^{5}$ The most common mode of poisoning is ingestion of the compound. ${ }^{8}$ Interestingly, we had cases with intramuscular injections, inhalational sprays. Pesticide poisoning being a very common presentation in the ED, this topic is not widely dealt in literatures of our part of world. Though being a major preventable cause of morbidity, there is no exact documented data on this issue. It was necessary to have a complete profile of the poisoning cases inour context so that better patient care can be provided. This study was designed with the objective to have a complete clinico-epidemiological profile with an in hospital outcome study of all the pesticide poisoning cases presenting to BPKIHS admitted under the department of Internal Medicine. Based on these observations a prospective study on the outcome of OP poisoning and importance of $10 \mathrm{PM}$ notes is started at BPKIHS.

\section{METHODS}

The study was designed as an observational study. All the patients with poisoning were classified broadly on the basis of the type of compound ingested. For the classification of cases International Statistical Classification of Diseases and Related Health Problems (ICD) was used. The study was conducted at B.P.Koirala Institute of Health Sciences, Dharan, Nepal and cases admitted during the period from January 2005 till December 2010 were taken. There were 2621 cases of poisoning admitted under the department of Internal Medicine. Of all the cases, 1661 were included in study for analysis. The cases of poisoning which were confirmed due to pesticides were only taken. All the cases were admitted from the emergency department after getting the initial management to the different units of medical wards. The diagnosis was made on the basis of definite history of ingestion of pesticide and clinical features and appropriate ICD code was given at the time of discharge. Most of time, the patient party brought the container. When the compound used was not clear an atropine challenge test was done and if positive then patient was classified as poisoning due to organophosphate and managed accordingly. Resources for the estimation of plasma or blood cholinesterase level were not available at the hospital. The characteristic manifestations of OP compounds were noted and for other non OP pesticides the diagnosis was solely on the container or patients or their relatives information. The basic demographic data was collected. The compound ingested, dose of atropine and PAM was recorded in patients with ingestion of OP compounds and in cases of halogenated insecticides like organochlorines or phosphides the patient was admitted for close monitoring. All the patients had complete blood count, electrocardiography, blood gases, renal function test, chest radiography and electrolytes done. All the complications were recorded and final outcome noted. The data was entered into Microsoft Excel sheet 2007 and analysis was done using SPSS software version 11.5 .

\section{RESULTS}

All the patients presenting with pesticide poisoning from January 2005 till December 2010 were taken. Patients who used other agents for the poisoning were excluded. To avoid discrepancy only those patients who fell under the group T60 and one of its subgroup under the pesticides category of ICD-10 were taken for the analysis (table 1).

\begin{tabular}{|c|c|}
\hline ICD CODE & DESCRIPTION \\
\hline T60.0 & $\begin{array}{l}\text { Organophosphate and } \\
\text { carbamate insecticides }\end{array}$ \\
\hline T60.1 & $\begin{array}{l}\text { Halogenated insecticides } \\
\text { Excludes: chlorinated } \\
\text { hydrocarbons }\end{array}$ \\
\hline T60.2 & Other insecticides \\
\hline T60.3 & Herbicides and fungicides \\
\hline T60.4 & Rodenticides \\
\hline T60.8 & Other pesticides \\
\hline T60.9 & Pesticide, unspecified \\
\hline
\end{tabular}




\begin{tabular}{|c|c|c|c|}
\hline \multicolumn{4}{|c|}{$\begin{array}{l}\text { Table 2. Socio-demographic characteristics of } \\
\text { patients with Pesticide poisoning. }\end{array}$} \\
\hline \multicolumn{2}{|c|}{ Characteristics Categories } & $\begin{array}{l}\text { No. of } \\
\text { patients }\end{array}$ & Percentage \\
\hline \multirow{3}{*}{ Age in years } & $15-40$ & 1391 & 83.74 \\
\hline & $41-65$ & 248 & 14.94 \\
\hline & $>65$ & 22 & 1.32 \\
\hline \multicolumn{2}{|c|}{ Mean age in years \pm SD } & \multicolumn{2}{|c|}{$29.15 \pm 0.30$} \\
\hline \multirow[t]{3}{*}{ Sex } & Male & 741 & 44.6 \\
\hline & Female & 919 & 55.3 \\
\hline & $\leq 5$ days & 870 & 52.38 \\
\hline \multirow{3}{*}{$\begin{array}{l}\text { Hospital stay } \\
\text { in days }\end{array}$} & 6-10 days & 499 & 30.04 \\
\hline & $11-15$ days & 176 & 10.6 \\
\hline & >15 days & 116 & 6.98 \\
\hline \multicolumn{2}{|c|}{$\begin{array}{l}\text { Mean hospital stay in days } \\
\pm \text { SD }\end{array}$} & \multicolumn{2}{|c|}{$6.79 \pm 0.17$} \\
\hline \multirow{7}{*}{$\begin{array}{l}\text { Outcome of } \\
\text { patients }\end{array}$} & Improved & 1348 & 81.16 \\
\hline & $\begin{array}{l}\text { Death in } 48 \\
\text { hrs }\end{array}$ & 110 & 6.6 \\
\hline & $\begin{array}{l}\text { Death after } \\
48 \text { hrs }\end{array}$ & 58 & 3.54 \\
\hline & LAMA & 100 & 6 \\
\hline & Absconded & 10 & 0.6 \\
\hline & Referred & 4 & 0.2 \\
\hline & Others & 31 & 1.9 \\
\hline
\end{tabular}

Among the 2621 patients with poisoning it was found that $1661(63.37 \%)$ of the patients had consumed pesticide. The most common pesticide was organophosphate and carbamate compounds. Among the pesticides group $10.96 \%$ had poisoning with rodenticides and others were organophosphate (67\%) and carbamates or halogenated insecticides. (Graph 1)

The baseline characteristics of the patients are given in (table 2). The mean age of the patients was 29 years. The incidence was more in females with the male: female ratio of $1: 1.23$. About $28 \%$ of the patients were from Jhapa district followed by $24 \%$ from Sunsari and $22 \%$ from Morang districts.

The mean hospital stay was 6.7 days. The majority of patients $81.16 \%$ showed improvement whereas $6.6 \%$ of patients died within 24 hours of admission and $3.54 \%$ after 48 hours of admission. Among all the patients $0.5 \%$ patients were given ICU care and all others were managed in the different units of Medicine ward.

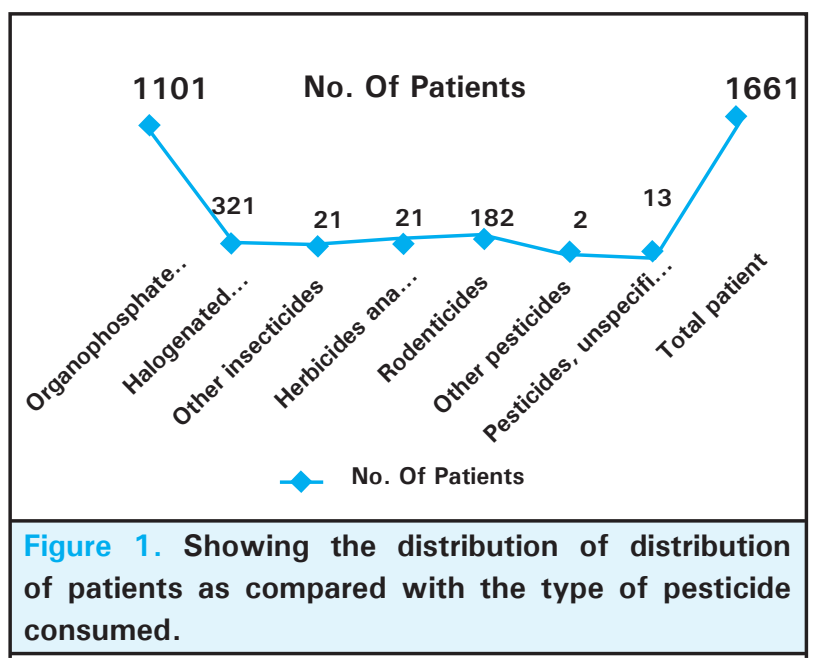

It was observed that there is an increasing incidence in the cases of poisoning though in the $206 /$ there was a decrease in the cases of pesticide poisoning presenting to our hospital. This can be due to other medical facilities which recently became available for the treatment. (Graph 2)

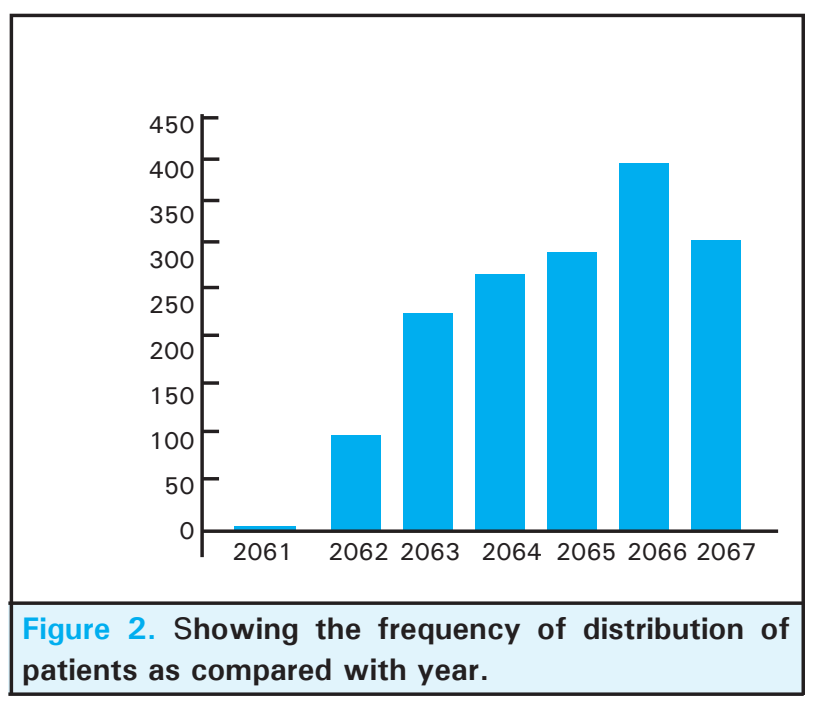

The most common mode of poisoning was ingestion. One of the patient had injected an OP compound in both his deltoids as an act of deliberate self harm where as some patients sprayed the pesticides in their mouth/ nose directly. There were 56 cases (5\%) of patients who had Intermediate syndrome. The patients who had consumed mixed compound (Chlorpyriphos and Cypermethrin) had increased chances of Intermediate syndrome. The total amount of atropine administered varied considerably from patient to patient, according to the need. Pralidoxime dose was $2 \mathrm{gms}$ intravenous every eight hourly infusion for 3-5 days. It was used in all the patients with organophosphorous poisoning 
who required atropinisation. There was one patient with OP Induced Delayed Polyneuropathy who was on physiotherapy but later lost to follow up as we could not trace data using the ID no. assigned to the patient even on OPD records. The ECG findings showed Sinus tachycardia, Prolongation of QTc interval, ST-T changes, Ventricular arrhythmias. Seven patients with organophosphorous poisoning had ST segment elevation. Zinc Phosphide related cardiotoxicity was seen in 6 patients which was lethal. The distribution of mortality and related pesticide is shown in (graph 3).

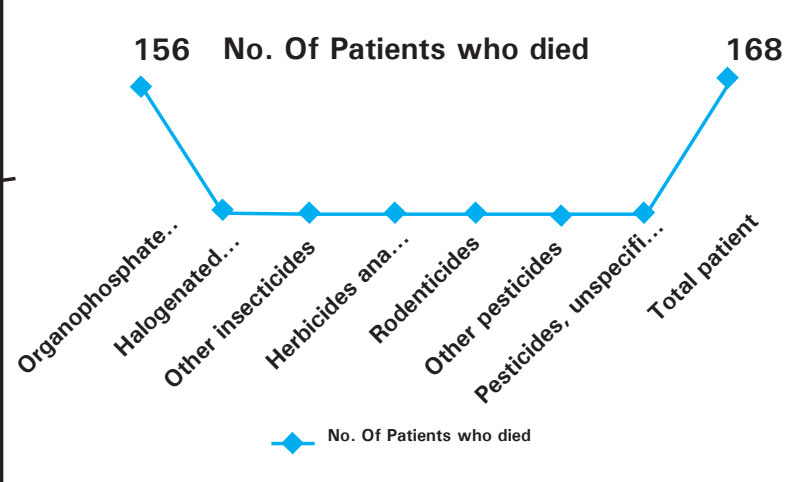

Figure 3. Showing the distribution of patients who died as compared to the type of compound ingested.

\section{DISCUSSION}

In this study the clinco-epidemiological profile of the patients with pesticide poisoning hasbeen shown. In a study done by the Nepal Drug and Poison Information Center, Kathmandu clearly demonstrated that the suicidal poisoning is a major public health problem. ${ }^{9}$ The most common age group for poisoning was 15-40 years $(83.7 \%)$. In another study from Nepal the most common age group was $15-25$ years $(50.1 \%)^{10}$ whereas in another study the mean age was 27 years. ${ }^{11}$ Male: Female ratio in this study was 1:1.23 which was similar in other studies from Nepal. Most of the poisoning cases were preceded be a quarrel and under the influence of alcohol. The most common substance used was organophosphates and carbamates $(66.3 \%)$ followed by organochlorines $(19.3 \%)$ then rodenticides $(11 \%)$ whereas in another study organophosphates (40\%) were most common agent followed by Phosphides (15 $\%) .{ }^{10}$ The reasons for this is easy availability of the Pesticides. Policy makers have not seriously realized the harmful effects of pesticides. According to the law, it is mandatory for a person or firm to acquire a certificate of registration before the import, export, sale or purchase of pesticides, insecticides and herbicides. But in practice the sale of pesticides still takes place openly without following these guidelines in many parts of the country. ${ }^{12}$ All the patients with organophosphorous poisoning who required atropinisation were given PAM. The dose used was according to the WHO recommendations. ${ }^{13}$ The study was not powered to see the effect of treatment on the outcome so the data on use of PAM and atropine could not be generalized and also there may be discrepancy on the dosing due to lack of fixed protocol among the various departments and units regarding the management of poisoning. After this retrospective study we started a prospective study looking at the requirement of the dose of atropine and PAM on the outcome. It was done as per a fixed protocol in the department of Internal Medicine.

The most common cause for referral or left against medical advice was the unavailability of intensive care unit beds. We did not have outcome data on these patients because there is no referral center nearby. The patients had to go to city in India if they required intensive care when it could not be arranged. The overall mortality in our study was $10.1 \%$ whereas the mortality in another study was $4.5 \% .^{10}$ The reasons may be due to delayed referral from other health care facilities and lack of intensive care beds. The mean hospital stay was 6.7 days. We also observed that cardiotoxicity in patients with Phosphide poisoning is lethal. All the patients with cardiotoxicity died. This lethality with phosphides has been shown in another study from Nepal. ${ }^{10}$ This may be due to unavailability of specific antidote.

Intermediate syndrome was seen in 5\% of the patients of OP poisoning. The intermediate syndrome is also known as type II paralysis. In a study done in India the incidence of intermediate syndrome was $29.4 \% .{ }^{14}$ This syndrome typically occurs after 1-4 days of exposure to $\mathrm{OP}$ poison but may occur even in the subsequent week. ${ }^{15}$ This difference may be due to the difference in the local availability of the compound. Another reason may be the difference in the documentation between the treating doctors. It has been shown in a study that organophosphorus insecticide poisoning is not a single entity;it is with substantial variability in clinical course, response to oximes, and outcome. Each organophosphorus insecticide should be considered as an individual poison and, consequently, patients might benefit from management protocols developed for particular organophosphorus insecticides. ${ }^{13}$ The study could not classify the severity of poisoning due to unavailability of the serum measurement of cholinesterase levels. We did not use the clinical severity scores to avoid inter-observer variations and the delay in the initial presentation. Also most of the patients were referred from primary health centers and sub-optimally atropinised when reached the emergency at BPKIHS. 


\section{CONCLUSIONS}

Pesticide poisoning is a major public health problem in Nepal. The incidence of poisoning is increasing. It affects mainly the young population. Females are at more risk to poisoning. Alcohol is a major contributor for ingestion of poison. Increasing indebtedness, fear of failing in different aspects, stress and meme of the society are major reasons to suicide. The mortality in patients with OP compounds is high even if antidotes are available. Cardiotoxicity is lethal. There should be implementation of strict law in the sale of the pesticides. The occupational hazards related to the compound should be studied and a large nationwide study should also be done on this aspect.

\section{Recommendations}

We recommend a strong law on the sale of these compounds. We recommend that there should be a fixed protocol in management of pesticide poisoning based on the WHO guidelines till further recommendations are available.We also recommend increasing the number of ICU beds and developing a protocol for triaging the patients regarding the ICU admissions.

\section{REFERENCES}

1. Rehiman S, Lohani SP and Bhattarai MD. Correlation of serum cholinesterase level, clinical score at presentation and severity of organophosphorous poisoning. JNMA 2008; 47(170):47-52.

2. Paudyal BP.Organophosphorus poisoning. JNMA 2008; 47(172):251-8.

3. Joshi S, Biswas B and Malla G. Management of organophosphorus poisoning. Update in anaesthesia.pg.1-5.

4. Eddleston M, Buckley NA, Eyer $\mathrm{P}$ and Dawson $\mathrm{AH}$. Management of acute organophosphorus pesticide poisoning.The Lancet 2008; 371:587-607.

5. Karki P, Ansari JA, Bhandary S and Koirala S. Cardiac and electrocardiographical manifestations of acute organophosphate poisoning. Singapore Med J 2004 Vol. 45(8): 385-389.

6. Haywood PT and Karalliedde L. Management of Poisoning due to organophosphorous compounds.Current anaesthesia \& Critical care 2000; 11:331-337.

7. Nepal in figures. Central beureau of satistics 2010.

8. Budhathoki S, Poudel P, Shah D, Bhatta NK, Dutta AK, Shah GS et al. Clinical profile and outcome of children presenting with poisoning or intoxication: a hospital based study. Nepal Med Coll J 2009; 11(3): 170-175.
9. Lohani SP. An epidemiological study of poisoning cases reported to the Nepal drug and poison information center, Kathmandu, Nepal.

10. Pokhrel D, Pant S, Pradhan A and Mansoor S. Comparative retrospective study of poisoning cases in central, zonal and district hospitals.Kathmandu university journal of science, engineering and technology2008; I (V): 40-48.

11. Kar SM, Timsinha S and Agrawal P.An Epidemiological study of Organophosphorus Poisoning at Manipal Teaching Hospital, Pokhara, Nepal. J Indian Acad Forensic Med: 32(2).p.108-109.

12. International POPs Elimination Project Fostering Active and Efficient Civil Society Participation in Preparation for Implementation of the Stockholm Convention Governmental and Public Awareness-raising on POPs Forum for Justice Nepal. February 2006:page 1-8.

13. Eddleston M, Eyer P, Worek F, Mohamed F, Senarathna L, BuckleyN et al. Differences between organophosphorus insecticides in humaself-poisoning: aprospective cohort study. The Lancet:Vol 366 October 22, 2005.

14. Palimar V, Arun M, Kumar M and Saralaya KM. Intermediate syndrome in organophosphorous poisoning.JIAFM, 2005 : 27 (1).28-30.

15. Goel Ashish and Agrawal Praveen. Pesticide Poisoning. The National Medical Journal Of India Vol. 20(4);2007. 